\title{
Trichinella spiralis
}

National Cancer Institute

\section{Source}

National Cancer Institute. Trichinella spiralis. NCI Thesaurus. Code C125965.

A species of parasitic nematodes in the genus Trichinella that is found worldwide. There are four region-specific subspecies that have different carnivorous animal hosts.

Trichinosis infection may occur through the ingestion of raw or undercooked animal tissue that contains encysted larvae. The larvae invade intestinal mucosa, where sexual maturation occurs. Female nematodes penetrate the mucosa and larvae travel through the bloodstream to penetrate and encyst in muscle tissue. 